\title{
Nucleosynthesis constraints on active-sterile neutrino conversions in the early universe with random magnetic field
}

\author{
V.B. Semikoz ${ }^{1}$, J.W.F. Valle ${ }^{2}$ \\ Instituto de Física Corpuscular - C.S.I.C., Departament de Física Teòrica, Universitat de València, 46100 \\ Burjassot, València, Spain
}

Received 25 February 1994; accepted 19 May 1994

\begin{abstract}
We consider active-sterile neutrino conversions in the early universe hot plasma in the presence of a random magnetic field generated at the electroweak phase transition. Within a random field domain the magnetization asymmetry of the lepton antilepton plasma produced by a uniform constant magnetic field is huge in contrast to their small density asymmetry, leading to a drastic change in the active-sterile conversion rates. Assuming that the random field provides the seed for the galactic field one can estimate the restrictions from primordial nucleosynthesis. Requiring that the extra sterile neutrino does not enter in equilibrium with the active ones before nucleosynthesis we find limits of the oscillation parameters which are stronger than in the isotropic case.
\end{abstract}

\section{Introduction}

Recent observations of cosmic background temperature anisotropies on large scales by the COBE satellite indicate the need for the existence of a hot dark matter (HDM) component, contributing about $30 \%$ of the total mass density, i.e. $\Omega_{\mathrm{HDM}} \sim 0.3$ [1]. Simple extensions of the standard electroweak model that can reconcile all known hints for neutrino masses, including solar and atmospheric neutrino observations postulate the

\footnotetext{
${ }^{1}$ On leave from the Institute of the Terrestrial Magnetism, the Ionosphere and Radio Wave Propagation of the Russian Academy of Sciences, IZMIRAN, Troitsk, Moscow region, 142092, Russia.

${ }^{2}$ E-mail: VALLE at vm.ci.uv.es or 16444::VALLE.
} 
existence of a light sterile neutrino $\nu_{s}[2,3]$. In some of these models such light sterile neutrino is the HDM candidate [2].

The most stringent constraints for the neutrino mass matrix including such a fourth kind of neutrino, a singlet $\nu_{s}$, are obtained from the nucleosynthesis bound on the maximum number of neutrino species ( $\Delta N \leqslant 0.3$ ) that can reach thermal equilibrium before nucleosynthesis and thus change the primordially produced helium abundance [4].

In an isotropic early Universe hot plasma such constraint on the additional neutrino species leads to an excluded region in the oscillation parameters $\left|\Delta m^{2}\right|, \sin ^{2} 2 \theta_{0}$ characterizing the active-sterile neutrino oscillations which can be estimated (in non-resonant case) as [5] ${ }^{3}$

$$
\begin{array}{ll}
\sin ^{4} 2 \theta_{0}\left|\Delta m^{2}\right| \gtrsim 5 \times 10^{-6} \mathrm{eV}^{2}, & \nu_{a}=\nu_{e} \\
\sin ^{4} 2 \theta_{0}\left|\Delta m^{2}\right| \gtrsim 3 \times 10^{-6} \mathrm{eV}^{2}, & \nu_{a}=\nu_{\mu, \tau} .
\end{array}
$$

In this paper we reconsider the active-sterile neutrino oscillation parameters assuming a new physical state of the hot ultrarelativistic plasma before nucleosynthesis $\left(T \gg m_{e}\right)$ with the inclusion of the random magnetic field hypothesis proposed in Ref. [8]. This random magnetic field could be generated at the electroweak phase transition near the temperature $T \sim T_{\mathrm{EW}}$ and could provide the seed for the galactic field in the dynamo enhancement mechanism [9]. In Refs. [10,11] this hypothesis was used in order to place stringent constraints on the Dirac neutrino magnetic moments.

In this paper we neglect neutrino magnetic moments, both diagonal as well as transition moments, and consider the magnetization asymmetry of the primordial early universe hot plasma produced by huge random magnetic fields. This influences the neutrino spectrum in the medium and modifies the neutrino conversions $\nu_{a} \leftrightarrow \nu_{s}$

We confine ourselves to a small random magnetic field domain size $L_{0}$, obeying the inequality $L_{0} \ll l_{\mathrm{H}}$. Within such a domain the magnetic field may be taken as uniform and constant, so that the magnetization of the plasma can be easily calculated. Here $l_{\mathrm{H}} \sim M_{\mathrm{Pl}} / T^{2}$ is the horizon length, $M_{\mathrm{Pl}}$ is the Planck mass and $T$ is the plasma temperature.

Although the magnetic field in different domains is randomly aligned relative to the neutrino propagation direction, we show how the observable neutrino conversion probabilities depend on the mean-squared random field via a squared magnetization value, therefore leading to nonvanishing averages over the magnetic field distribution. We apply this to the active-sterile neutrino conversions in order to obtain more stringent limits than those that apply in the absence of magnetic field.

\footnotetext{
${ }^{3}$ The discrepancy between these estimates and those Refs. [6,7] stems mainly from different estimates for the collision rate which the authors of Ref. [5] have evaluated in detail.
} 


\section{Dirac neutrino spectrum in a hot plasma with magnetic field}

In order to derive the Dirac equation for neutrino propagation in a uniform locally anisotropic medium characterized by a constant (within a domain) magnetic field $\boldsymbol{B}=$ $(0,0, B)$ we start from the effective four-fermion interaction Lagrangian of the standard electroweak theory

$$
\mathcal{L}_{\mathrm{int}}=G_{\mathrm{F}} \sqrt{2} \bar{\psi}_{\nu_{b}} \gamma^{\mu} \frac{\left(1-\gamma_{5}\right)}{2} \psi_{\nu_{b}} \sum_{a}\left\langle\bar{\psi}_{a}\left[c_{V}^{b a} \gamma_{\mu}-c_{A}^{b a} \gamma_{\mu} \gamma_{5}\right] \psi_{a}\right\rangle_{0} \ldots,
$$

where the indices $a, b=e, \mu, \tau$ correspond to the lepton generations, and the dots denote the extra terms including hadrons. Here $c_{V}^{b a}=2 \sin ^{2} \theta_{W} \pm 0.5$ is the vector coupling constant (upper sign for $a=b$ ), $\sin ^{2} \theta_{W}$ is the electroweak mixing parameter and $c_{A}^{b a}=\mp 0.5$ is the corresponding axial coupling constant (upper sign for $b=a$ ). Finally, the symbol $\langle\ldots\rangle_{0}$ denotes the statistical averaging of vector and axial vector currents, using equilibrium Fermi distributions $f_{\kappa^{\prime} \kappa}^{(a)}=\left\langle b_{\kappa}^{(a)+} b_{\kappa^{\prime}}^{(a)}\right\rangle_{0}=f_{\lambda^{\prime} \lambda}^{(a)}\left(p_{z}, n\right)$. Explicitly, these are given as

$$
f_{\kappa^{\prime} \kappa}^{(a)}=\frac{\delta_{\lambda \lambda^{\prime}}}{\exp \left[\left(\varepsilon_{n \lambda}\left(p_{z}\right)-\zeta_{a}\right) / T\right]+1},
$$

where the lepton spectrum is of the form

$$
\varepsilon_{n \lambda}\left(p_{z}\right)=\sqrt{p_{z}^{2}+m_{a}^{2}+|e| B(2 n+1-\lambda)} .
$$

For the case of antileptons, one has $f_{\kappa^{\prime} \kappa}^{(\tilde{a})}=\left\langle d_{\kappa}^{(a)+} d_{\kappa^{\prime}}^{(a)}\right\rangle_{0}=f_{-\lambda^{\prime},-\lambda^{\prime}}^{(a)}\left(p_{z}, n\right)$, or explicitly,

$$
f_{\kappa^{\prime} \kappa}^{(\tilde{a})}=\frac{\delta_{-\lambda,-\lambda^{\prime}}}{\exp \left[\left(\varepsilon_{n \lambda}\left(p_{z}\right)+\zeta_{a}\right) / T\right]+1},
$$

where the antilepton spectrum is of the form

$$
\varepsilon_{n \lambda}\left(p_{z}\right)=\sqrt{p_{z}^{2}+m_{a}^{2}+|e| B(2 n+1+\lambda)} .
$$

Here $\zeta_{a}$ is the chemical potential and the full set of the quantum numbers $\kappa$ includes $\left\{p_{z}, n, \lambda\right\}$, where $p_{z}$ is the conserved momentum component for the chosen magnetic field geometry; $n=0,1,2, \ldots$ is the Landau number and $\lambda= \pm 1$ is twice the eigenvalue of the conserved lepton spin projection on the magnetic field, $\left(\sigma_{z}\right)_{\lambda^{\prime} \lambda}=\lambda \delta_{\lambda^{\prime} \lambda}$. The change of sign $\lambda$ in Eq. (2.3) arises from the conjugation property $C \sigma_{i} C^{-1}=-\sigma_{i}^{T}$.

The resulting equation describing the neutrino motion takes the form

$$
\left[\hat{q}-m_{\nu_{b}}^{\mathrm{vac}}-V_{b}^{(\mathrm{vec})} \gamma_{0} \frac{\left(1-\gamma_{5}\right)}{2}-V_{b}^{(\text {axial })} \gamma_{z} \frac{\left(1-\gamma_{5}\right)}{2}\right]\left(\begin{array}{l}
\varphi \\
\chi
\end{array}\right)=0,
$$

where the vector interaction potential [12] of an active (left) neutrino $\nu_{b}(b=e, \mu, \tau)$, $V_{b}^{(\text {vec })}=G_{\mathrm{F}} \sqrt{2} \sum_{a} c_{V}^{(a b)}\left\langle\bar{\psi}_{a} \gamma_{0} \psi_{a}\right\rangle^{4}$ is given by the known formula [5]:

\footnotetext{
${ }^{4}$ Note that spatial components of the mean vector current are zero, $\left\langle\bar{\psi} \gamma_{i} \psi\right\rangle=0, i=1,2,3$.
} 


$$
V_{b}^{(\mathrm{vec})}=G_{\mathrm{F}} \sqrt{2} n_{\gamma}\left[L_{0}^{\left(v_{b}\right)}-A_{b} \frac{T^{2}}{M_{W}^{2}}\right] .
$$

The first term in the vector potential Eq. (2.6) that is proportional to the small particleantiparticle asymmetries $L_{\alpha}=\left(n_{\alpha}-n_{\tilde{\alpha}}\right) / n_{\gamma}$, normalized on the photon density $n_{\gamma}=$ $0.244 T^{3}$, is given by [5]

$$
L_{0}^{\left(\nu_{b}\right)}=\sum_{a} c_{V}^{b a} \times L_{a}+\left(1-4 \sin ^{2} \theta_{W}\right) L_{p} / 2-L_{n} / 2+2 L_{\nu_{b}}+\sum_{a \neq b} L_{\nu_{a}} .
$$

This term is changed a little due to the effect of the strong magnetic field on the charged lepton (antilepton) densities. However this change remains negligible compared with the non-local second term in Eq. (2.6) which, for the case of electron neutrinos, is given by

$$
\left|V_{\nu_{e}}^{(\mathrm{vec})}\right| \approx 3.4 \times 10^{-20}\left(\frac{T}{\mathrm{MeV}}\right)^{5} \mathrm{MeV}
$$

We have neglected the influence of the magnetic field on the nucleon densities, due to the very small magnetic moments of the nucleons and the small temperatures below the QCD phase transition $(T \lesssim 200 \mathrm{MeV})$.

Now we turn to the new axial term in the Dirac equation. This term is obtained from the basic underlying $S U(2) \otimes U(1)$ electroweak gauge theory Lagrangean describing neutrino interactions in the medium, by just taking the averaged matrix element of the relevant axial current in the medium, namely

$$
V_{b}^{(\text {axial })}=\frac{G_{\mathrm{F}}}{\sqrt{2}} \sum_{a=e, \mu, \tau}\left(-2 c_{A}^{b a}\right)\left\langle\bar{\psi}_{a} \gamma_{z} \gamma_{5} \psi_{a}\right\rangle_{0}
$$

Although this definition is sufficient for our purposes, it is useful to give an alternative interpretation of the effect of the magnetic field in terms of the relevant macroscopic concept, namely the magnetization asymmetry of each component $(a=e, \mu, \tau)$ of the hot plasma in the external magnetic field, we call it $M_{z}^{(a)}-M_{z}^{(\tilde{a})}$,

$$
M_{i}^{(a)}-M_{i}^{(\bar{a})}=\mu_{B}\left\langle\psi_{a}^{+} \Sigma_{i} \psi_{a}\right\rangle_{0} \equiv \mu_{B}\left\langle\bar{\psi}_{a} \gamma_{i} \gamma_{5} \psi_{a}\right\rangle_{0},
$$

where $\mu_{B}=|e| / 2 m_{a}$ is the Bohr magneton. Note that for our chosen magnetic field geometry only the $z$-component of the axial current is nonvanishing.

While the first definition is closer to particle physics notions and, to this extent more basic, we will find it useful to use both definitions interchangeably in what follows.

The new macroscopic axial term $V_{b}^{(\text {axial })}$ changes the active neutrino spectrum in the plasma. In the ultrarelativistic limit $m_{v_{b}} \rightarrow 0$ such spectrum can be obtained, from Eq. (2.5), as

$$
E=V_{b}^{(\mathrm{vec})}+\sqrt{q_{\perp}^{2}+\left(q_{z}+V_{b}^{(\mathrm{axial})}\right)^{2}}
$$

This differs from the isotropic one, $E=V_{b}^{(\mathrm{vec})}+q$, due to the shift of the $z$-component of the total neutrino momentum $q=\left(q_{\perp}^{2}+q_{z}^{2}\right)^{1 / 2}$. In the hot plasma the neutrino 
momentum $\langle q\rangle \sim 3 T$ is larger than both $V_{b}^{(\text {vec })}$ and $V_{b}^{(\text {axial })}$, so that we can use for neutrino oscillation problem the ultrarelativistic approximation,

$$
E \approx q+V_{b}^{(\mathrm{vec})}+V_{b}^{(\mathrm{axial})} \frac{q_{z}}{q}+\frac{\left(V_{b}^{(\mathrm{axial})}\right)^{2} q_{\perp}^{2}}{2 q^{3}} .
$$

\section{Magnetization asymmetry of hot plasma in an external magnetic field}

In order to show the importance of the axial term contribution Eq. (2.8) in the neutrino spectrum, let us consider the main features of the hot lepton-antilepton plasma in a uniform constant magnetic field $B=(0,0, B)$.

One can easily check that the lepton-antilepton density asymmetry $\left\langle\bar{\psi}_{a} \gamma_{0} \psi_{a}\right\rangle_{0}=n_{a}-n_{\tilde{a}}$ equals to

$$
n_{a}-n_{\tilde{a}}=\sum_{n=0}^{\infty} \frac{|e| B}{(2 \pi)^{2}} \int_{-\infty}^{\infty} d p_{z} \operatorname{Tr}\left[f^{(a)}\left(p_{z}, n\right)-f^{(\tilde{a})}\left(p_{z}, n\right)\right]
$$

where trace is calculated over spin variables $\lambda$ and the (-) sign inside Eq. (3.1) arises from the $N$-ordering of operators in the current $N\left(\bar{\psi}_{a} \gamma_{\mu} \psi_{a}\right)$.

In order to check the normalization of the Fermi distribution Eq. (2.1), let us consider the WKB approximation $n \gg 1$ in the weak magnetic field limit $|e| B \ll T^{2}$ changing $2|e| B n$ into $p_{\perp}^{2}$. Using $|e| B d n=p_{\perp} d p_{\perp}$ one obtains the standard isotropic result

$$
n_{a}-n_{\tilde{a}}=2 \int \frac{d^{3} p}{(2 \pi)^{3}}\left[\frac{1}{\exp \left(\left(\sqrt{p_{z}^{2}+p_{\perp}^{2}+m_{a}^{2}}-\zeta\right) / T+1\right)}-(\zeta \rightarrow-\zeta)\right],
$$

for the lepton-antilepton asymmetry Eq. (3.1). The factor "2" is produced by the spin sum. Note that the contribution of our general asymmetry Eq. (3.1) to the neutrino vector potential Eq. (2.6) is negligible comparing with the main non-local term Eq. (2.7).

Now we calculate with help of Eq. (2.1) the magnetization asymmetry in the hot plasma. In analogy with Eq. (3.1) we can write the lepton contribution to the magnetization asymmetry as

$$
M_{j}^{(a)}=\mu_{B} \sum_{n=0}^{\infty} \frac{|e| B}{(2 \pi)^{2}} \int_{-\infty}^{\infty} d p_{z} \operatorname{Tr}\left[\sigma_{j} f^{(a)}\left(p_{z}, n\right)\right]
$$

Using the trace $\operatorname{Tr}\left[\sigma_{j} f^{(a)}\right]=\sum_{\lambda \lambda^{\prime}}\left(\sigma_{j}\right)_{\lambda \lambda^{\prime}} f_{\lambda^{\prime} \lambda}^{(a)}\left(p_{z}, n\right)=\delta_{j z} \sum_{\lambda} \lambda f_{\lambda \lambda}^{(a)}\left(p_{z}, n\right)$ with $\left(\sigma_{z}\right)_{\lambda \lambda^{\prime}}=\lambda \delta_{\lambda \lambda^{\prime}}$ and $\delta_{\lambda \lambda}=1$ for $\lambda= \pm 1$, one can easily show that, due to the degeneracy of the Landau levels $n=1,2, \ldots, \varepsilon_{n+1,1}=\varepsilon_{n,-1}$ ) all terms in the sum

$$
\sum_{n=0}^{\infty} \frac{1}{\exp \left(\left(\varepsilon_{n, 1}-\zeta\right) / T\right)+1}-\frac{1}{\exp \left(\left(\varepsilon_{n,-1}-\zeta\right) / T\right)+1}
$$


cancel ${ }^{5}$ except for contribution of the main (non-degenerate) Landau level $(n=0)$

$$
\frac{1}{\exp \left(\left(\varepsilon_{0,1}-\zeta\right) / T\right)+1}=\frac{1}{\exp \left(\left(\sqrt{p_{z}^{2}+m_{a}^{2}}-\zeta\right) / T\right)+1}
$$

Finally, the lepton part becomes

$$
M_{z}^{(a)}=\mu_{B} \frac{2|e| B}{(2 \pi)^{2}} \int_{0}^{\infty} d p_{z}\left[\frac{1}{\exp \left(\left(\sqrt{p_{z}^{2}+m_{a}^{2}}-\zeta\right) / T\right)+1}\right] .
$$

Now we turn to the antilepton part. Using the $C$-conjugation of the relevant operators we must add to the trace in the integrand of Eq. (3.2) the trace over negative spin projections

$$
-\sum_{\lambda, \lambda^{\prime}}\left(-\sigma_{j}^{T}\right)_{-\lambda^{\prime} ;-\lambda} f_{-\lambda ;-\lambda^{\prime}}^{(\tilde{a})}\left(p_{z}, n\right),
$$

where the (-) sign before the sum arises from the $\mathrm{N}$-ordering of the operators in the axial current $N\left(\bar{\psi}_{a} \gamma_{\mu} \gamma_{5} \psi_{a}\right)$, and the second one comes from the $C$-conjugation property $C \sigma_{j} C^{-1}=-\sigma_{j}^{T}$. Now one can easily show, by using the change $\lambda \rightarrow-\lambda$, that the antilepton part may be rewritten as

$$
\begin{aligned}
& -\sum_{\lambda \lambda^{\prime}}\left(-\sigma_{j}\right)_{\lambda^{\prime} ; \lambda} f_{\lambda \lambda^{\prime}}^{(\tilde{a})}\left(p_{z}, n\right) \\
& =-\sum_{\lambda \lambda^{\prime}} \frac{(-\lambda) \delta_{\lambda^{\prime} \lambda} \delta_{\lambda \lambda^{\prime}}}{\exp \left(\left(\sqrt{p_{z}^{2}+m_{a}^{2}+|e| B(2 n+1-\lambda)}+\zeta\right) / T\right)+1},
\end{aligned}
$$

where we used the conserved spin $z$-component eigenvalue $\left(\sigma_{z}\right)_{\lambda^{\prime} \lambda}=+\lambda \delta_{\lambda^{\prime} \lambda}$. Similarly as obtained above for the lepton part, all of the Landau-level contributions $n=1,2 \ldots$. cancel due to the degeneracy property $\varepsilon_{n+1,1}=\varepsilon_{n,-1}$. Thus the magnetization asymmetry is obtained by summing lepton and antilepton contributions, in contrast to the density asymmetry,

$$
\begin{aligned}
M_{z}^{(a)}-M_{z}^{(\tilde{a})}= & \mu_{B} \frac{2|e| B}{(2 \pi)^{2}} \int_{0}^{\infty} d p_{z}\left[\frac{1}{\exp \left(\left(\sqrt{p_{z}^{2}+\bar{m}_{a}^{2}}-\zeta\right) / T\right)+1}\right. \\
& \left.+\frac{1}{\exp \left(\left(\sqrt{p_{z}^{2}+m_{a}^{2}}+\zeta\right) / T\right)+1}\right]
\end{aligned}
$$

\footnotetext{
${ }^{5}$ One can easily check that in the weak magnetic field limit $|e| B \ll T^{2}$, due to the subtraction of the Fermi distribution functions with different spin projections, from the relativistic magnetization Eq. (3.2) one recovers the known result corresponding to the spin paramagnetism of the non-relativistic free electron gas in a metal [13]: $M_{z}=-2 \mu_{B}^{2} B \int D(E) \frac{d f}{d E} d E$. Note that here we used the isotropic phase volume with the energy $E=p^{2} / 2 m_{e}, D(E)=\left(2 m_{e}\right)^{3 / 2} \sqrt{E} /(2 \pi)^{2}$ and that the Fermi distribution in non-relativistic case is given by $f(E)=\left[\exp \left(\left(E-\zeta^{\prime}\right) / T\right)+1\right]^{-1}$ where the chemical potential is $\zeta^{\prime}=\zeta-m_{e}$.
} 
Substituting this result into the axial potential in Eq. (2.8) and Eq. (2.9) one can finally rewrite the ultrarelativistic $\left(m_{a}=\zeta=0\right.$ ) neutrino spectrum Eq. (2.11) as

$$
E=q+V_{b}^{(\mathrm{vec})}+f(q, B)
$$

where the magnetic field contribution $f(q, B)=V_{b}^{(\text {axial })} q_{z} / q+\left(V_{b}^{(\text {axial })}\right)^{2} q_{\perp}^{2} / 2 q^{3}$ is given by

$$
f(q, B)=\mu_{\mathrm{eff}} \frac{\boldsymbol{q} \boldsymbol{B}}{q}+\frac{\mu_{\mathrm{eff}}^{2}}{2 q}\left(B^{2}-\frac{(\boldsymbol{q} \boldsymbol{B})^{2}}{q^{2}}\right)
$$

and the quantity $\mu_{\text {eff }}$ is defined by ${ }^{6}$

$$
\mu_{\mathrm{eff}}=\frac{e G_{\mathrm{F}}\left(-2 c_{A}\right) T \ln 2}{\sqrt{2} \pi^{2}} \approx 6 \times 10^{-13} \mu_{B}\left(\frac{T}{\mathrm{MeV}}\right) .
$$

For a hot plasma ( $T \gg 1 \mathrm{MeV}$ ) this is huge, in contrast to the small lepton-antilepton density asymmetry Eq. (3.1). This arises from the fact that the magnetization asymmetry is produced by the mean axial current so that the lepton and antilepton contributions add instead of subtract. In a strong uniform magnetic field the first term in Eq. (3.8) may exceed the non-local term Eq. (2.7) considered by Notzold and Raffelt. As we will show below, for large random magnetic fields, such term can drastically change the active-sterile neutrino conversion rates.

\section{Active-sterile neutrino conversions in a hot plasma with random magnetic field}

Let us now consider the wave equation describing the propagation of a system of active (doublet) and light sterile (singlet) neutrinos, with masses $m_{1}$ and $m_{2}$, mixing angle $\theta$, and no transition magnetic moments, in the presence of a random magnetic field. We postulate the following evolution equation ${ }^{7}$ :

$$
\begin{aligned}
& i \frac{d}{d t}\left(\begin{array}{l}
\nu_{d} \\
\nu_{s}
\end{array}\right) \\
& \quad=\left[\begin{array}{cc}
\left(c^{2} m_{1}^{2}+s^{2} m_{2}^{2}\right) / 2 q+V_{d}+f(q, B) & c s \Delta \\
s c \Delta & \left(s^{2} m_{1}^{2}+c^{2} m_{2}^{2}\right) / 2 q
\end{array}\right]\left(\begin{array}{l}
\nu_{d} \\
\nu_{s}
\end{array}\right),
\end{aligned}
$$

where we use the standard definitions $\Delta=\Delta m^{2} / 2 q ; \Delta m^{2}=m_{2}^{2}-m_{1}^{2} ; c=\cos \theta$, and $s=\sin \theta$. In addition we have denoted by $V_{d}$ the vector part of the active neutrino potential of Eq. (2.6).

\footnotetext{
${ }^{6}$ Note that this effective magnetic moment has no relation with the real anomalous neutrino magnetic moment which we neglect.

${ }^{7}$ Strictly speaking, in order to describe the active to sterile neutrino conversions one has to start from a system of two majorana neutrinos and not from the Dirac equation as we did in Section 2 Eq. (2.5). This can be done and one finds [14] that our ansatz is obtained in the ultrarelativistic small mixing angle limit.
} 
In the phase of the early universe hot plasma of interest to us we have only active neutrinos in thermal equilibrium. From Eq. (4.1) one can easily obtain a nonlinear integro-differential equation for the conversion probability $P_{\nu_{d} \rightarrow \nu_{s}}(t)$ from active to sterile neutrinos, $\nu_{d} \rightarrow \nu_{s}$. It obeys the unitarity condition

$$
P_{\nu_{d} \rightarrow \nu_{s}}(t)=\left\langle\nu_{s}^{*} \nu_{s}\right\rangle=1-P_{\nu_{d} \rightarrow \nu_{d}}(t)=\left\langle\nu_{d}^{*} \nu_{d}\right\rangle
$$

Defining $P_{\nu_{d} \rightarrow \nu_{s}} \equiv P(t)$ and averaging over the ensemble of random magnetic fields one obtains:

$$
\frac{d^{2} P}{d t^{2}}+\Delta_{m}^{2} P+\left\langle\Delta_{B}^{2}\right\rangle \int_{0}^{t} d t_{1} K\left(t-t_{1}\right) \frac{d P\left(t_{1}\right)}{d t_{1}}=\frac{\Delta^{2} \sin ^{2} 2 \theta}{2},
$$

where the initial conditions are given by

$$
P(0)=\dot{P}(0)=0 .
$$

The factor before the second term,

$$
\Delta_{m}^{2}=\left(V_{d}-\Delta \cos 2 \theta\right)^{2}+\Delta^{2} \sin ^{2} 2 \theta
$$

is the well known oscillation squared frequency in an isotropic hot plasma [5]. Due to the property of randomness $\left\langle B_{z}\right\rangle=0$ the factor before integral term in Eq. $(4.2),\left\langle\Delta_{B}^{2}\right\rangle=$ $2\left(V_{d}-\Delta \cos 2 \theta\right)\langle f(q, B)\rangle+\left\langle f^{2}(q, B)\right\rangle$, with the function $f(q, B)$ from Eq. (3.8), is determined mainly by the second term $\left\langle\Delta_{B}^{2}\right\rangle \approx\left\langle f^{2}(q, B)\right\rangle$. For collisionless neutrino propagation along z-axis $q=(0,0, q)$, the factor $\left\langle\Delta_{B}^{2}\right\rangle$ takes of the form

$$
\left\langle\Delta_{B}^{2}\right\rangle \simeq \mu_{\mathrm{eff}}^{2}\left\langle\boldsymbol{B}^{2}\right\rangle / 3,
$$

after averaging over random magnetic fields.

Here the effective neutrino magnetic moment is given by Eq. (3.9) with the mean squared first term of Eq. (3.8) given by $\left\langle B_{z}^{2}(t)\right\rangle=\left\langle\boldsymbol{B}^{2}\right\rangle / 3$. Note that the second term contribution can be neglected in the ultrarelativistic limit. Finally the kernel in Eq. (4.2) $K\left(t_{1}-t_{2}\right)=\left\langle B_{z}\left(t_{1}\right) B_{z}\left(t_{2}\right)\right\rangle /\left\langle B_{z}^{2}(t)\right\rangle$ depends on the model of random fields. If we choose the simple model with uncorrelated magnetic field domains of the same small size $L_{0}{ }^{8}, K\left(t_{1}-t_{2}\right)=L_{0} \delta\left(t_{1}-t_{2}\right)$, the integro-differential equation Eq. (4.2) reduces to a second order differential equation with the boundary conditions Eq. (4.3). The solution of this equation is of the form

\footnotetext{
${ }^{8}$ For neutrinos crossing many domains $t=L \gg L_{0}$ the size $L_{0}$ corresponds to the width of the narrow resonance $L_{0}^{2} /\left(L_{0}^{2}+t^{2}\right)$ for our assumed $\delta$-correlated random fields

$$
\frac{K(t)}{L_{0}} \sim \lim _{L_{0} \rightarrow 0} \frac{L_{0}}{t^{2}+L_{0}^{2}}=\frac{\pi}{2} \delta(t)
$$
}




$$
\begin{aligned}
& P(t)=\frac{\Delta^{2} \sin ^{2} 2 \theta}{2 \Delta_{m}^{2}}\{1-\exp (-\Gamma t) \\
& \left.\quad \times\left[\cosh \left(\sqrt{\Gamma^{2}-\Delta_{m}^{2}} t\right)+\frac{\Gamma}{\sqrt{\Gamma^{2}-\Delta_{m}^{2}}} \sinh \left(\sqrt{\Gamma^{2}-\Delta_{m}^{2}} t\right)\right]\right\},
\end{aligned}
$$

where

$$
\Gamma=\frac{\left\langle\Delta_{B}^{2}\right\rangle L_{0}}{2}
$$

is the damping parameter of neutrino oscillations in a random field. In the weak magnetic field limit $\Gamma \ll \Delta_{m}$, where the neutrino oscillation frequency in matter $\Delta_{m}$ is given by Eq. (4.4), such solution,

$$
P(t) \simeq \frac{\Delta^{2} \sin ^{2} 2 \theta}{2 \Delta_{m}^{2}}\left(1-\exp (-\Gamma t) \cos \Delta_{m} t\right),
$$

reproduces the known case of $\nu_{d} \leftrightarrow \nu_{s}$ oscillations in an isotropic hot plasma if $\Gamma=0$. For strong random magnetic fields generated at the electroweak phase transition [8] the opposite condition $\Gamma \gg \Delta_{m}$ is fulfilled. The corresponding asymptotics of the solution Eq. (4.6),

$$
P(t) \simeq \frac{\Delta^{2} \sin ^{2} 2 \theta}{2 \Delta_{m}^{2}}\left(1-\exp \left(-\Delta_{m}^{2} t / 2 \Gamma\right)\right),
$$

is mostly aperiodic, in contrast to Eq. (4.8). From Eq. (4.9) one define the relaxation time $t_{\text {relax }} \sim 2 \Gamma / \Delta_{m}^{2}=\left\langle\Delta_{B}^{2}\right\rangle L_{0} / \Delta_{m}^{2}$. The condition that the neutrino crosses many domains, leads to the requirement $\left\langle\Delta_{B}^{2}\right\rangle \gg \Delta_{m}^{2}$. In the next section we verify that this condition is indeed fulfilled.

\section{Random magnetic fields before nucleosynthesis}

In order to show the validity of Eq. (4.9) let us estimate the factor $\left\langle\Delta_{B}^{2}\right\rangle$ in Eq. (4.5) in formula Eq. (4.7) substituting to Eq. (4.5) the mean squared random magnetic field

$$
\sqrt{\left\langle B^{2}\right\rangle}=10^{24} G\left(\frac{T}{T_{\mathrm{EW}}}\right)^{2} \times\left(\frac{L_{0}}{L}\right)^{p}
$$

with the scale dependence obeying the index $p=1 / 2[15]$.

Requiring that the primordial magnetic field survives beyond the recombination epoch leads to a minimal domain size [16],

$$
L_{0} \gtrsim 10^{4} \mathrm{~cm}\left(T_{B B N} / T\right) \sim 10^{3} \mathrm{~cm}(\mathrm{MeV} / \mathrm{T}) .
$$

With this assumption let us now estimate a lower limit for Eq. (4.5). In order to do this we use the collisionless neutrino propagation approximation, i.e. $t=L \leqslant t_{\text {coll }}=\Gamma_{W}^{-1}$, and substitute Eq. (5.2) into Eq. (5.1) leading to 


$$
\left\langle\Delta_{B}^{2}\right\rangle^{1 / 2}=\frac{\mu_{\mathrm{eff}}\left\langle B^{2}\right\rangle^{1 / 2}}{\sqrt{3}} \gtrsim 6 \times 10^{-17}\left(\frac{T}{\mathrm{MeV}}\right)^{5} \mathrm{MeV}
$$

Note that this is significantly larger than the nonlocal vector potential term given in Eq. (2.7). Therefore, we have obtained a self-consistent requirement $\left\langle\Delta_{B}^{2}\right\rangle \gg \Delta_{m}^{2}$ which justifies our use of the $\delta$-correlated random magnetic fields.

If instead we put into Eq. (5.1) the maximum scale $L=l_{\mathrm{H}}(T)$, where $l_{\mathrm{H}}$ is the horizon length at temperature $T$ we obtain another estimate $\left\langle\Delta_{B}^{2}\right\rangle^{1 / 2} \gtrsim 2 \times 10^{-16}(T / \mathrm{MeV})^{3.5} \mathrm{MeV}$, which also exceeds Eq. (2.7). However, in such case one should include the effect of collisions on the neutrino conversions.

Substituting the limit Eq. (5.2) rewritten as $L_{0} \gtrsim\left(10^{14} / 1.9\right)(\mathrm{MeV} / \mathrm{T}) \mathrm{MeV}^{-1}$ and the estimate in Eq. (5.3) into Eq. (4.7) we see that random magnetic field Eq. (5.1) obeys the condition $\Gamma \gg \Delta_{m} \sim\left|V_{d}\right|$. This demonstrates the validity of our main approximation to the conversion probability Eq. (4.9).

\section{Nucleosynthesis bounds on the sterile neutrino conversions}

Comparing the relaxation time in the probability Eq. (4.9) estimated with help of Eq. (2.7), Eq. (4.4), Eq. (5.2) and Eq. (5.3) as

$$
t_{\text {relax }} \sim 2 \Gamma / \Delta_{m}^{2} \gtrsim 2 \times 10^{20} T^{-1}
$$

with the collision time,

$$
t_{\text {coll }} \sim 2 \times 10^{21}(\mathrm{MeV})^{4} \mathrm{~T}^{-5},
$$

one finds a critical temperature which separates two regimes $T_{*} \sim 10^{1 / 4} \mathrm{MeV}$ above which it should be important to take into account collisions. For times less than the neutrino collision time $t_{\text {coll }}=\Gamma_{W}^{-1}$ we can directly estimate the sterile neutrino conversion probability of Eq. (4.9) in the collisionless approximation. It is also very simple to consider the alternative limit where one can average over many collisions. In both cases one obtains essentially the same result. Finally, for the case of intermediate temperatures close to $T_{*} \sim 2 \mathrm{MeV}$ one needs a more accurate kinetic approach as in Ref. [5].

For definiteness, we consider here the regime the derivation of the nucleosynthesis bounds for active-sterile neutrino oscillations in the case where one can average over many collisions. Averaging $t_{\text {coll }}^{-1} \int_{0}^{t c o l l} d t P(t)$ from Eq. (4.9) one obtains the result

$$
\langle P\rangle_{\text {coll }}=\frac{\Delta^{2} \sin ^{2} 2 \theta}{2 \Delta_{m}^{2}}\left[1-\frac{2 \Gamma \Gamma_{W}}{\Delta_{m}^{2}}+\frac{2 \Gamma \Gamma_{W}}{\Delta_{m}^{2}} \exp \left(-\frac{\Delta_{m}^{2}}{2 \Gamma \Gamma_{W}}\right)\right] .
$$

The factor in brackets above, $f(x)=1-x^{-1}+\exp (-x) / x$, where $x=\Delta_{m}^{2} / 2 \Gamma \Gamma_{W}$, is a monotonic function which attains its minimum value $f(0)=0$ as $\Gamma \rightarrow \infty$ and tends asymptotically to $f(x=\infty)=1$ when one neglects the magnetic field, i.e. $\Gamma \rightarrow 0$. In a strong random magnetic field this factor is restricted by $f\left(x_{\max }\right)$, when we substitute the estimates Eq. (5.2) and Eq. (5.3) into the damping factor $\Gamma$ in Eq. (4.7),

$$
\Gamma \geq \Gamma_{\min } \approx 10^{-19}(T / \mathrm{MeV})^{9} \mathrm{MeV}
$$


or

$$
x \lesssim x_{\max }=\frac{\Delta_{m}^{2}}{2 \Gamma_{W} \Gamma_{\min }} \approx 10\left(\frac{T}{\mathrm{MeV}}\right)^{-4}
$$

and $f(x) \lesssim f\left(x_{\max }\right)$. In Eq. (6.5) we have used the neutrino non-resonant oscillation frequency estimate $\Delta_{m} \sim V_{d}$, where $V_{d}$ is given by the Nötzold-Raffelt result Eq. (2.7) and the usual weak interaction rate $\Gamma_{W}=4.0 G_{\mathrm{F}}^{2} T^{5} \sim 5.4 \times 10^{-22}(T / \mathrm{MeV})^{5} \mathrm{MeV}$.

One can easily see from Eq. (6.5) that for temperatures $T \gtrsim 3 \mathrm{MeV}$ the argument $x$ is very small, $x \ll 1$, so we can rewrite the probability Eq. (6.3) as

$$
\langle P\rangle_{\mathrm{coll}}=\frac{\sin ^{2} 2 \theta_{B}}{4 L_{0} I_{W}}
$$

where the mixing angle in the the hot plasma with magnetic field is given by $\sin ^{2} 2 \theta_{B}=$ $\Delta^{2} \sin ^{2} 2 \theta /\left\langle\Delta_{B}^{2}\right\rangle$ and is restricted due to Eq. (5.3) $(\langle q\rangle \sim 3 T)$ by

$$
\sin ^{2} 2 \theta_{B} \simeq \frac{\sin ^{2} 2 \theta\left(\Delta m^{2}\right)^{2}}{36 T^{2}\left(\Delta_{B}^{2}\right)} \lesssim 10^{7}\left(\frac{\Delta m^{2}}{\mathrm{eV}^{2}}\right)^{2}\left(\frac{T}{\mathrm{MeV}}\right)^{-12} \sin ^{2} 2 \theta
$$

Taking into account Eq. (5.2), we find that the sterile neutrino production rate $\Gamma_{\nu_{s}}(t \leqslant$ $\left.t_{\text {coll }}\right)=P \Gamma_{W}$ obtained from Eq. (6.6), $\Gamma_{\nu_{s}}=\sin ^{2} 2 \theta_{B} / 4 L_{0}$, obeys the inequality

$$
\Gamma_{\nu_{s}} \lesssim \frac{1}{2} \times 10^{-7}\left(\frac{\Delta m^{2}}{\mathrm{eV}^{2}}\right)^{2} \sin ^{2} 2 \theta\left(\frac{T}{\mathrm{MeV}}\right)^{-11} \mathrm{MeV} .
$$

Sterile neutrinos would be thermalized if this rate Eq. (6.8) exceeds the Hubble expansion rate $H=4.5 \times 10^{-22}(T / \mathrm{MeV})^{2} \mathrm{MeV}$,

$$
\Gamma_{\nu_{s}} / H \geq 1
$$

Using the inequality Eq. (6.8) we obtain a new constraint on the $\left|\Delta m^{2}\right|, \sin 2 \theta$ oscillation parameters,

$$
\left|\Delta m^{2}\right||\sin 2 \theta| \lesssim 10^{-7} \times\left(\frac{T}{\mathrm{MeV}}\right)^{13 / 2} \mathrm{eV}^{2}
$$

Note that the sign of $\Delta \mathrm{m}^{2}$ for us here is irrelevant, in contrast to the isotropic case.

Self-consistency of our approximations (see Eq. (6.5)) requires us to assume in Eq. (6.9) a minimal temperature $T \gtrsim T_{\min } \sim 3 \mathrm{MeV}$ which then allows us to rewrite Eq. (6.9) as

$$
\left|\Delta m^{2}\right||\sin 2 \theta| \lesssim 10^{-4} \mathrm{eV}^{2}
$$

We see that this bound can be significantly stronger than the nonresonant estimate Eq. (1.1) obtained for the case of an isotropic hot plasma (see Fig. 1). This is especially so for the case of small mixing angle ( $\sin 2 \theta \sim 0.1$ ), where we obtain from Eq. (6.10) an excluded region of the squared mass difference $\left|\Delta m^{2}\right| \gtrsim 10^{-3} \mathrm{eV}^{2}$ instead of the result of Eq. (1.1), $\left|\Delta m^{2}\right| \gtrsim 5 \times 10^{-2} \mathrm{eV}^{2}$. 


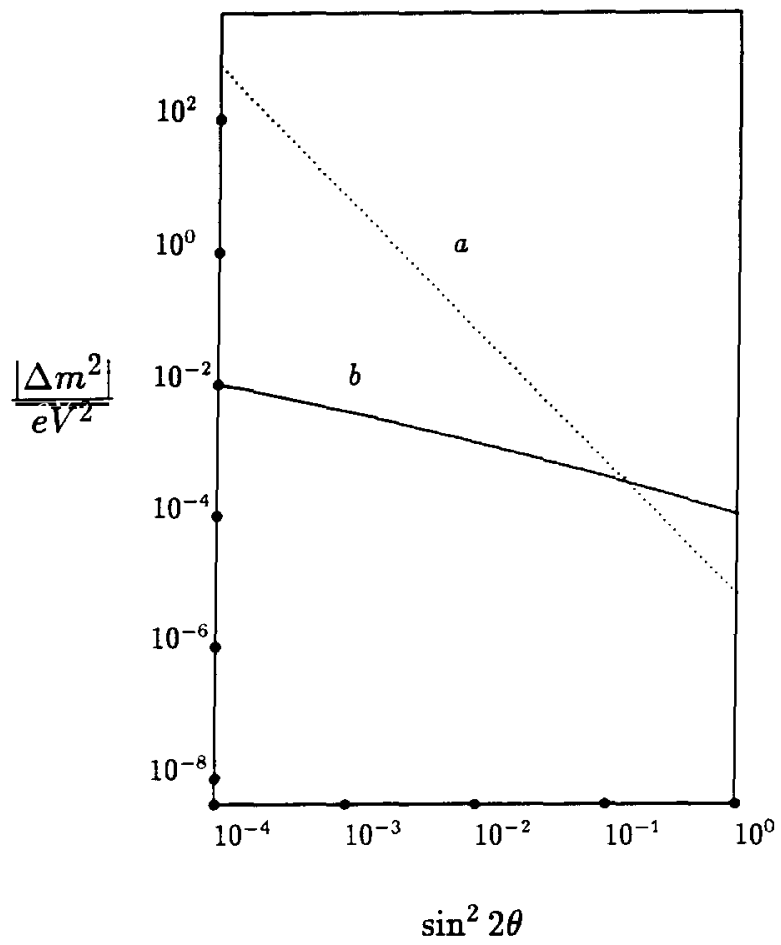

Fig. 1. Regions of $\nu_{e} \leftrightarrow \nu_{s}$ oscillation parameters excluded by nucleosynthesis: (a) the region above the dotted line is excluded by the requirement that $N_{\nu}<3.4$ for the non-resonant isotropic hot plasma estimate of Ref. [5]; (b) the region above the solid line is excluded in present work for the the case a hot plasma with primordial random magnetic field, seed for the galactic field, by the requirement that $N_{\nu}<4$ for $T \gtrsim T_{\min } \sim 3 \mathrm{MeV}$, Eq. (6.10).

Note also that, in the case of the MSW resonance, $\left(\Delta_{m}^{2}=\Delta^{2} \sin ^{2} 2 \theta\right.$, see Eq. (4.4), the argument $x=\Delta_{m}^{2} / 2 \Gamma \Gamma_{W}$ in the probability Eq. (6.3) is less than

$$
x \lesssim x_{\max }=3 \times 10^{14}\left(\frac{\Delta m^{2}}{\mathrm{eV}^{2}}\right)^{2} \sin ^{2} 2 \theta\left(\frac{T}{\mathrm{MeV}}\right)^{-16} .
$$

If the upper limit here is much less than unity, we automatically obtain from Eq. (6.3) the same probability Eq. (6.6) that does not depend on the frequency $\Delta_{m}$ at all. Therefore, the constraint Eq. (6.9) would be a general one and substituting acceptable values of the product $\left(\Delta m^{2} / \mathrm{eV}^{2}\right)^{2} \sin ^{2} 2 \theta \lesssim 10^{-14}(\mathrm{~T} / \mathrm{MeV})^{13}$ into Eq. (6.11) we confirm the validity of the ultrarelativistic approximation $x \lesssim x_{\max } \lesssim 3 \times(T / \mathrm{MeV})^{-3} \ll 1$ for this resonant regime of $\nu_{e} \leftrightarrow \nu_{s}$ oscillations too.

\section{Discussion and conclusions}

The existence of huge magnetic fields generated at the electroweak phase transition modifies the neutrino spectrum in the early universe hot plasma. This happens due to the 
magnetization of each component of the plasma. Within a small random magnetic field domain the local uniform constant magnetic field approximation allows us to calculate the magnetization, which is proportional to the random magnetic field.

In contrast to the small lepton antilepton density asymmetry produced by the mean vector current the magnetization asymmetry produced by the mean axial current is large, due to their opposite charge conjugation properties. As a result the lepton and anti lepton magnetizations add instead of subtract.

Averaging the differential equation describing the evolution of the $\nu_{d} \rightarrow \nu_{s}$ conversion probabilities over the random magnetic field distribution we find a nonvanishing mean squared field contribution which drastically changes the conversion rates with respect to those of the isotropic case.

Assuming that the primordial magnetic fields generated at the electroweak phase transition are the origin of the observed galactic fields and requiring that there should be no more than one extra neutrino species in equilibrium before nucleosynthesis we derive new and more stringent constraints on the active-sterile neutrino oscillation parameters than in the isotropic case without random magnetic field. In contrast to what happens in the isotropic case, our constraints do not depend on the active-sterile neutrino conversion channel beyond the obvious dependence contained in $\Delta \mathrm{m}^{2}$.

The fact that the constraints can be stronger in our case than in the isotropic case, despite the fact that the conversion rates is smaller, follows from the different way in which these conversion rates depend on the oscillation parameters. In particular, in the random field case there is a more sensitive dependence of the average conversion rates upon the neutrino squared mass difference. While in the isotropic case there is a saturation of this probability as a function of the neutrino squared mass difference, in our case we have a linear dependence upon $\Delta \mathrm{m}^{2}$.

\section{Acknowledgements}

This work was supported by DGICYT under grant number PB92-0084, and partially by an european grant CHRX-CT93-0132 and by a senior researcher NATO fellowship (V.S.). We thank Dr. A.I. Rez who pointed out the simple solution of Eq. (4.2).

\section{References}

[1] G.F. Smoot et al., Astrophys. J. 396 (1992) L1-L5.

[2] J.T. Peltoniemi, D. Tommasini and J.W.F. Valle, Phys. Lett. B 298 (1993) 383-390.

[3] J.T. Peltoniemi and J.W.F. Valle, Nucl. Phys. B 406 (1993) 409-422.

[4] T. Walker, G. Steigman, D.N. Schramm, K. Olive and H. Kang, Astrophys. J. 376 (1991) 51.

[5] A.D. Dolgov, Sov. J. Nucl. Phys. 33 ( 1981) 700;

L. Stodolsky, Phys. Rev. D 36 (1987) 2273;

M.A. Rudsky, Astrophys. Space Sci. 165 (1990) 65;

K. Enqvist, K. Kainulainen and M.J. Thomson, Nucl. Phys. B 373 (1992) 498;

G. Raffelt, G. Sigl and L. Stodolsky, Phys. Rev. Lett. 70 (1993) 2363.

[6] K. Kainulainen, Phys. Lett. B 224 (1990) 191.

[7] R. Barbieri and A. Dolgov, Phys. Lett. B 237 (1990) 440; Nucl. Phys. B 349 (1991) 742;

J. Cline, Phys. Rev. Lett. 68 (1992) 3137 
[8] T. Vachaspati, Phys. Lett. B 265 (1991) 258-261.

[9] P. Olesen, Phys. Lett. B 281 (1992) 300.

[10] K. Enqvist, P. Olesen and V. Semikoz, Phys. Rev. Lett. 69 (1992) 2157.

[11] K. Enqvist, V. Semikoz, A. Shukurov and D. Sokoloff, Phys. Rev. D 48 (1993) 4557.

[12] D. Nötzold and G. Raffelt, Nucl. Phys. B 307 (1988) 924.

[13] R. Kubo and T. Nagamiya, Solid State Physics, Mc'Grau Book Co. Inc., New York - Oxford - Toronto (1969) p. 491.

[14] S. Pastor, V. Semikoz and J.W.F. Valle, Valencia preprint FTUV-94/14 and paper in preparation

[15] K. Enqvist and P. Olesen, Phys. Lett. B 319 (1993) 178.

[16] B. Cheng, D.N. Schramm and J.W. Truran, Preprint Fermilab-Pub-93-259-A. 